\title{
The relation between serum levels of epidermal growth factor and necrotizing enterocolitis in preterm neonates
}

\author{
Heba Mostafa Ahmed, MD', Nsreen Mostafa Kamel, MD² \\ Departments of ${ }^{1}$ Pediatrics and ${ }^{2}$ Clinical and Chemical Pathology, Faculty of Medicine, Beni-Suef University, Beni-Suef, Egypt
}

Purpose: Necrotizing enterocolitis (NEC) is one of the most serious complications of prematurity. Many risk factors can contribute to the development of NEC. The epidermal growth factor (EGF) plays a major role in intestinal barrier function, increases intestinal enzyme activity, and improves nutrient transport. The aim of this study was to assess the role of epidermal growth factor in the development of NEC in preterm neonates.

Methods: In this study, 130 preterm neonates were included and divided into 3 groups, as follows: group 1, 40 preterm neonates with NEC; group 2, 50 preterm neonates with sepsis; and group 3, 40 healthy preterm neonates as controls. The NEC group was then subdivided into medical and surgical NEC subgroups. The serum EGF level was measured using enzyme-linked immunosorbent assay.

Results: Serum EGF levels ( $\mathrm{pg} / \mathrm{dL}$ ) were significantly lower in the NEC group (median [interquartile range, IQR], 9.6 [2-14]) than in the sepsis (10.1 [8-14]) and control groups (11.2 [8-14], $P<0.001$ ), with no significant difference between the sepsis and control groups, and were positively correlated with gestational age $(r=0.7, P<0.001)$. A binary logistic regression test revealed that low EGF levels and gestational ages could significantly predict the development of NEC. The receiver-operating characteristic curve for EGF showed an optimal cutoff value of $8 \mathrm{pg} / \mathrm{mL}$, with $73.3 \%$ sensitivity, $98 \%$ specificity, and an area under the curve of 0.92 .

Conclusion: The patients with NEC in this study had significantly lower serum EGF levels $(P<0.001)$, which indicated that EGF could be a reliable marker of NEC in preterm neonates.

Key words: Epidermal growth factor, Neonates, Necrotizing enterocolitis

\section{Introduction}

Necrotizing enterocolitis (NEC) is one of the most serious complications of prematurity, with a prevalence of 7\% in very low birth weight infants and mortality between 20\% and 30\%. ${ }^{1)}$ The high rate of morbidity and mortality is due to the multifactorial pathogenesis of NEC. There is no a clear mechanism involved as a cause of NEC, only several factors that contributes to it. ${ }^{2}$ These factors include undeveloped gastrointestinal mucosal barrier, immature innate and humoral immunity, uncoordinated GIT movement, and the overgrowth of pathogenic bacteria. ${ }^{3)}$ There are many risk factors can contribute to the development of NEC as prematurity, formula feeding, hypoxic-ischemic insult, and abnormal bacterial colonization of the gut. Yet, no single risk factor is crucial, and the mechanisms by which they precipitate NEC are mostly unclear.4) Initial management of NEC is generally supportive and includes stopping enteric feedings and providing total parenteral nutrition, continuous nasogastric suctioning with intestinal decompression, and administration of broad-spectrum antibiotics. ${ }^{5} \mathrm{NEC}$ occurs with variety of severity ranging from a mild form that improves with antibiotics and discontinuation of
Corresponding author: Heba Mostafa Ahmed, $\mathrm{MD}$

Departments of Pediatrics, Faculty of Medicine, Beni-Suef University, Mohammed Hassan street, Beni-Suef governate, Egypt

Tel: +201001516641

Fax: +201001516641

E-mail: heba_most@yahoo.com

https://orcid.org/0000-0002-8086-3759

Received: 22 September, 2018

Revised: 1 February, 2019

Accepted: 14 March, 2019
Copyright (c) 2019 by The Korean Pediatric Society

This is an open-access article distributed under the terms of the Creative Commons Attribution NonCommercial License (http://creativecommons.org/ licenses/by-nc/4.0/) which permits unrestricted noncommercial use, distribution, and reproduction in any medium, provided the original work is properly cited. 
oral feedings (medical NEC) to a severe form that leads to intestinal perforation, peritonitis, and eventually death. ${ }^{6)}$ Approximately 20\%-40\% of all infants diagnosed with NEC require surgical intervention. ${ }^{7)}$ Many studies had have attempted to find out biomarkers for the early detection of NEC. ${ }^{8)}$ Epidermal growth factor (EGF) is a $50-\mathrm{kDa}$ multifunctional protein that has antiangiogenic, antitumorigenic and neurotrophic functions. ${ }^{9}$ It belongs to a vast family of peptides (transforming growth factor-alpha, amphiregulin, heparinbinding EGF, epiregulin, betacellulinn and neuroregulin, among others) which provoke a variety of biological responses in the gastrointestinal tract, the majority of which involve regulation of cell replication and cell movement and survival. Also, it enhances the proliferation and differentiation of epithelial cells. ${ }^{10}$

The aim of this study was to assess the relation between EGF serum level and the development of NEC in preterm neonates.

\section{Materials and methods}

This study was conducted between March 2015 and April 2017. One hundred thirty preterm neonates were included in the study and were divided into 3 groups; group 1 (40 preterms with NEC), group 2 (50 preterms with sepsis), and group 3 (40 healthy preterms as normal controls). Patient inclusion was established by the presence of signs specific for NEC by modified Bell's criteria ${ }^{11)}$ or, for the sepsis group, by either clinical evidence of sepsis and/or positive blood cultures. Preterms with history of previous abdominal surgery, or a known congenital anomaly of the gastrointestinal tract or abdominal wall were excluded from the study. All included subjects were subjected to (1) full history taking stressing on gestational age (GA), sex, obstetrical history (mode of delivery), obstetrical problems placenta abnormalities, premature rupture of membranes, etc., (2) clinical examination including: general examination, local, physical examination and measurements (weight, length), and vital signs. Laboratory investigations included C-reactive protein (CRP), complete blood picture with differential counts, blood culture, sensitivity, and serum EGF levels. Erect abdominal X-ray and abdominal ultrasonography were done to cases with NEC then NEC group was divided accordingly to 2 subgroups (surgical NEC and medical NEC). To perform the multiple regression tests the included neonates are divided into 2 groups; group 1 patients without NEC, group 2 patients with NEC

The local ethical committee of Beni-Suef University Hospital approved the study protocol (approval number: 187-36) and a written consent was obtained from caregivers of the included neonates.

\section{Group definitions}

(1) NEC group: preterm neonates fulfilling at least stage IIA modified Bell's criteria; (2) Surgical NEC group: patients with radiographic evidence of pneumoperitoneum or physiologic deterioration despite maximal medical therapy that necessitates laparotomy; (3) Sepsis group: preterms with the clinical evidence of sepsis (poor activity, temperature instability etc.) with high CRP levels and positive blood picture without evidence of NEC according to modified Bell's criteria; (4) Control group: Healthy preterms who did not require incubator admission or admitted without clinical or laboratory evidence of sepsis.

\section{Samples collection}

A volume of $3 \mathrm{~mL}$ of venous blood was drawn from each neonate under complete aseptic conditions and divided into 2 tubes. The first tube contained EDTA for evaluating complete blood cell and the second tube was left in the incubator for 30 minutes, centrifuged at 3,000 rpm for 10 minutes and then the separated serum was stored at $-80^{\circ} \mathrm{C}$ till the end of the study to evaluate the other laboratory parameters. EGF was assayed by using EGF ELISA Kit (E-EL-H1875, Elabscience Biotechnology Inc., Houston, TX, USA).

\section{Statistical methods}

Statistical analysis was done using IBM SPSS Statistics ver. 22.0 (IBM Co., Armonk, NY, USA). Data were described in the form of median and interquartile range (IQR) for quantitative data, and frequency and proportions for qualitative data. A $P$ value $<0.05$ was considered statistically significant. Differences were analyzed between the groups by nonparametric test (median test for $\mathrm{k}$ samples). Binary logistic regression test was used to determine the relation between variables. Correlations were analyzed by Spearman correlation coefficient test. The receiver operating characteristics (ROC) curve analysis was performed to identify the optimal cutoff value for EGF level distinguishing between the cases with NEC and sepsis.

\section{Results}

There was no difference between the groups as regarding gender and GA. Age and body weight were significantly higher in control group than sepsis and NEC groups but no difference between sepsis and NEC groups. Hemoglobin levels and TLC were statistically insignificant between the 3 groups while NEC group had lower platelets levels than other groups. CRP was significantly higher in sepsis and NEC groups as compared to controls. EGF was significantly lower in the sera of NEC group (median [IQR], 9.6 [2-14]) as compared to both sepsis (median [IQR], 10.1 [8-14]) and control (median [IQR], 11.2 [8-14]) groups and there was no difference between control and sepsis groups (Table 1). Blood culture was positive in 100\% of cases with sepsis while it was positive in $87 \%$ in NEC group. Sixty-two percent (25 cases) of NEC group had medical NEC while surgical NEC was reported in 38\% (15 cases). On comparing medical and surgical NEC groups; GA, age, and body weight were significantly lower in surgical NEC group. EGF levels were lower in surgical NEC but it 
Table 1. Demographic and laboratory data of the study groups

\begin{tabular}{|c|c|c|c|c|}
\hline Variable & Control & Sepsis & NEC & $P$ value \\
\hline GA (day) & 238 (210-245) & 231 (196-245) & 238 (210-245) & 0.2 \\
\hline Age (day) ${ }^{a)}$ & 8 (2-25) & $17(8-27)$ & $12(7-25)$ & 0.002 \\
\hline Weight $(\mathrm{kg})^{\mathrm{a})}$ & $1.71(0.90-3.50)$ & $1.23(0.90-2.90)$ & $1.50(0.95-3.10)$ & $<0.001$ \\
\hline $\mathrm{Hb}(\mathrm{g} / \mathrm{L})$ & $13.31(10-18.80)$ & $12.71(7.11-17.62)$ & $12.22(7.11-16.72)$ & 0.08 \\
\hline $\operatorname{TLC}\left(\times 10^{3} / \mathrm{cm}^{3}\right)$ & $11.00(3.50-33.20)$ & $16.22(3.50-28.50)$ & $12.21(3.60-33.20)$ & 0.16 \\
\hline Neutrophils \% & 53 (32-69) & $48(30-68)$ & $64(35-82)$ & 0.009 \\
\hline Lymphocytes \% & 38.51 (22-78) & $42(16-63)$ & 27 (9-59) & 0.001 \\
\hline Platelets $\left(\times 10^{3} / \mu \mathrm{L}\right)$ & 265 (200-402) & $200(168-620)$ & $80(24-143)$ & $<0.001$ \\
\hline $\mathrm{CRP}(\mathrm{mg} / \mathrm{dL})$ & $0(0-6)$ & $48(12-96)$ & $48(12-96)$ & $<0.001$ \\
\hline EGF (pg/dL) & $11.21(8.00-14.00)$ & $10.13(8.00-14.00)$ & $9.63(2.00-14.00)$ & $<0.001$ \\
\hline
\end{tabular}

Values are presented as median (interquartile range).

NEC, necrotizing enterocolitis; GA, gestational age; Hb, hemoglobin; TLC, total leukocytic counts; CRP, C-reactive protein; EGF, epidermal growth factor.

${ }^{a)} \mathrm{Age}$ and weight at the time of blood sampling.

Boldface indicates a statistically significant difference with $P<0.05$.

Table 2. Comparison between the surgical and medical NEC groups

\begin{tabular}{lccc}
\hline Variable & Surgical NEC & Medical I NEC & $P$ value \\
\hline Gestational age (wk) & $221(210-245)$ & $238(224-245)$ & 0.003 \\
Age (day) $)^{\text {a) }}$ & $10(9-17)$ & $14(7-25)$ & 0.33 \\
Weight $(\mathrm{kg})^{\text {a) }}$ & $1.22(0.95-2.11)$ & $1.54(1.02-3.11)$ & 0.04 \\
EGF $(\mathrm{pg} / \mathrm{dL})$ & $6.41(2.22-11.00)$ & $7.6(2.0-14.0)$ & 0.13 \\
\hline
\end{tabular}

Values are presented as median (interquartile range).

NEC, necrotizing enterocolitis; EGF, epidermal growth factor.

${ }^{\text {a) }}$ Age and weight at the time of blood sampling.

Boldface indicates a statistically significant difference with $P<0.05$.

Table 3. Binary logistic regression models for determining the relationships of EGF, sex, and gestational age with the development of NEC

\begin{tabular}{lcrrrrc}
\hline Variable & Coefficient & SE & Wald & $d f$ & $P$ value & $95 \% \mathrm{Cl}$ \\
\hline (Constant) & -23.359 & 11.889 & 3.860 & 1 & 0.049 & - \\
EGF & -1.300 & 0.320 & 16.568 & 1 & 0.000 & $0.14-0.51$ \\
Sex & -0.293 & 0.836 & 0.123 & 1 & 0.726 & $0.14-3.8$ \\
Gestational age & -0.149 & 0.057 & 6.779 & 1 & 0.009 & $1.03-1.29$
\end{tabular}

The dependent variable is group $(0=N O N E C, 1=N E C)$.

EGF, epidermal growth factor; NEC, necrotizing enterocolitis; SE, standard error; $d f$, degree of freedom; $\mathrm{Cl}$, confidence interval.

was statistically insignificant (Table 2). Binary logistic regression test was run to predict NEC based on EGF, GA, and sex. These variables statistically significantly predicted NEC, $\chi^{2}(3,130)=63.5, P<0.001$, $R^{2}=0.75$. There was a significant negative association between both EGF and GA as predictors of the development of NEC $(\beta=-1.3, P<$ $0.001)$ and $(\beta=-0.14, P=0.009)$ respectively. Sex has no influence on the prediction of development of NEC (Table 3). There was strong significant positive correlation between EGF and GA $(r=0.7, P<0.001)$ (Fig. 1). ROC curves were used to define the possible value of serum EGF level below which NEC is highly suspected to occur. The area under the ROC curve of serum EGF levels was 0.92 (95\% confidence interval, 0.83-0.97). The analysis rendered an optimal cutoff value

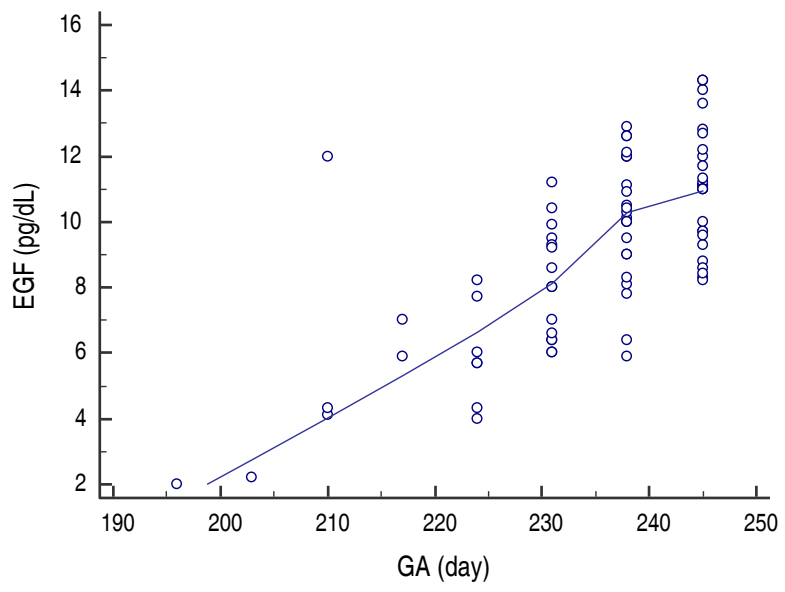

Fig. 1. Correlation between epidermal growth factor (EGF) levels and gestational age (GA). A significant positive correlation is observed between the EGF serum levels and gestational age, with the highest levels achieved at 230 days (33 weeks) of gestation.

of $8 \mathrm{pg} / \mathrm{mL}$ corresponding to $73.3 \%$ sensitivity and 98\% specificity with positive predictive value (PV) of 95\% and negative PV of 86.7 $\%$ (Fig. 2).

\section{Discussion}

The aim of this study was to assess the relation between EGF serum level and the development of NEC in preterm neonates and we found that patients with NEC had significantly lower serum levels of EGF indicating that EGF could be a reliable predictor of NEC in preterm neonates. The ultimate course of NEC cannot be predicted based on the clinical features alone so, efforts has been done for the identification of early biomarkers for NEC. ${ }^{11)} \mathrm{EGF}$ is a protein secreted by the salivary glands, kidneys, liver, Brunner's 


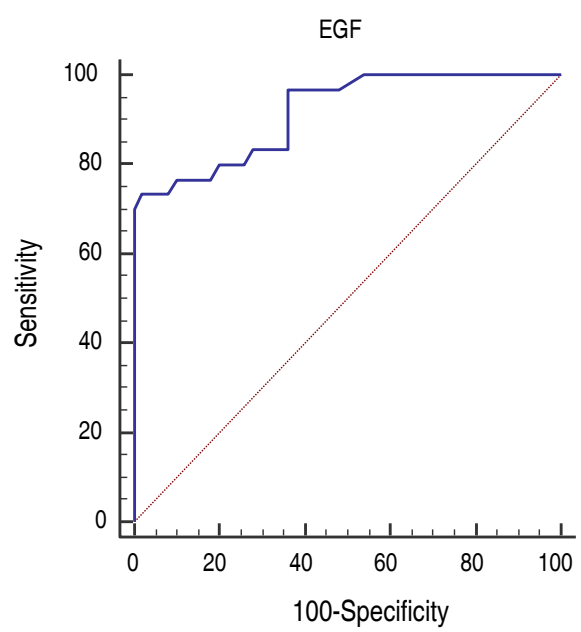

Fig. 2. Receiver-operating characteristic curve for the epidermal growth factor (EGF) cutoff value for the prediction of the development of necrotizing enterocolitis.

glands of the duodenum, and Paneth cells of the small intestine. ${ }^{12)}$ EGF stimulates a variety of biologic responses, such as cell replication, cell movement, and cell survival. ${ }^{13)}$ EGF plays a major role in the intestinal barrier function, increases intestinal enzyme activity, and improves nutrient transport. ${ }^{14)}$ Many experimental studies had demonstrated the role of EGF as a regulator of epithelial paracellular permeability ${ }^{15)}$ and goblet cell and mucin production in the intestinal epithelium. ${ }^{16)}$ EGF also acts as a bacterial colonization inhibitor. ${ }^{17)}$ In our study, we found that patients with NEC had significantly lower serum levels of EGF than the other 2 groups indicating the critical role of EGF in maintaining the integrity and function of the intestinal wall and its deficiency contributes to the development of NEC in preterm newborns. Meanwhile, the absence of a significant difference between the sepsis group and healthy controls proves that EGF is not just a marker of a proinflammatory state and it is a specific marker for NEC. In addition, we found that EGF and GA had a significant negative association with NEC indicating that low EGF levels and smaller GA are significant risk factors for the development of NEC. Shin et al. ${ }^{18)}$ reported in their study that premature neonates with NEC have diminished levels of EGF in serum and saliva compared with healthy controls. The emerging need for early detection of NEC for early management and prevention of complications makes EGF a good marker for early detection of NEC. Despite statistically insignificant, the lower levels of EGF in surgical NEC group make it also a possible indicator for the course of the disease and disease severity. Our finding of strong positive correlation between EGF level and GA is in agreement with Hofmann and Abramowicz ${ }^{19)}$ who found that amniotic fluid contains significant concentrations of EGF that gradually increase during pregnancy, with the highest level achieved at the end of the normal gestation period explaining the association between NEC and prematurity. In this study, results showed that, using a cutoff value of $<8 \mathrm{pg} / \mathrm{mL}$, serum EGF level can be used to predict the development of NEC with an AUC of 0.92 and an optimized sensitivity of 73.3\% and specificity of $98 \%$ with positive PV of 95\% making EGF a reliable biomarker for the diagnosis of NEC.

The main strength of this study is that we have included patients with a relatively larger number of preterm neonates suffering of NEC as compared to some previous studies

Our study has some limitations. First, no comparison was made between EGF and other inflammatory markers (as erythrocyte sedimentation rate and procalcitonin). Second, single measurement of EGF levels was done, so serial measurement of EGF might be more useful.

In conclusion, our study highlights the role of EGF in NEC. EGF may be considered a reliable marker for NEC that could help in identifying diseased neonates. A value lower than $8 \mathrm{pg} / \mathrm{L}$ should raise the suspicion of NEC.

\section{Conflicts of interest}

No potential conflict of interest relevant to this article was reported.

\section{References}

1. Caplan MS, Jilling T. New concepts in necrotizing enterocolitis. Curr Opin Pediatr 2001;13:111-5.

2. Neu J, Walker WA. Necrotizing enterocolitis. N Engl J Med 2011;364: 255-64.

3. Stey A, Barnert ES, Tseng CH, Keeler E, Needleman J, Leng M, et al. Outcomes and costs of surgical treatments of necrotizing enterocolitis. Pediatrics 2015;135:e1190-7.

4. Lin PW, Nasr TR, Stoll BJ. Necrotizing enterocolitis: recent scientific advances in pathophysiology and prevention. Semin Perinatol 2008; 32:70-82.

5. Cho SX, Berger PJ, Nold-Petry CA, Nold MF. The immunological landscape in necrotising enterocolitis. Expert Rev Mol Med 2016;18:e12.

6. Bradshaw WT. Necrotizing enterocolitis: etiology, presentation, management, and outcomes. J Perinat Neonatal Nurs 2009;23:87-94.

7. Stoll BJ, Hansen NI, Bell EF, Walsh MC, Carlo WA, Shankaran S, et al. Trends in care practices, morbidity, and mortality of extremely preterm neonates, 1993-2012. JAMA 2015;314:1039-51.

8. Young C, Sharma R, Handfield M, Mai V, Neu J. Biomarkers for infants at risk for necrotizing enterocolitis: clues to prevention? Pediatr Res 2009;65(5 Pt 2):91R-97R.

9. Filleur S, Nelius T, de Riese W, Kennedy RC. Characterization of PEDF: a multi-functional serpin family protein. J Cell Biochem 2009;106: 769-75.

10. Dvorak B, Halpern MD, Holubec H, Williams CS, McWilliam DL, Dominguez JA, et al. Epidermal growth factor reduces the development of necrotizing enterocolitis in a neonatal rat model. Am J Physiol Gastrointest Liver Physiol 2002;282:G156-64.

11. Kliegman RM, Walsh MC. Neonatal necrotizing enterocolitis: pathogenesis, classification, and spectrum of illness. Curr Probl Pediatr 
1987;17:213-88.

12. Playford RJ, Wright NA. Why is epidermal growth factor present in the gut lumen? Gut 1996;38:303-5.

13. Harris RC, Chung E, Coffey RJ. EGF receptor ligands. Exp Cell Res 2003;284:2-13.

14. Guo J, Sheng G, Warner BW. Epidermal growth factor-induced rapid retinoblastoma phosphorylation at Ser780 and Ser795 is mediated by ERK1/2 in small intestine epithelial cells. J Biol Chem 2005;280: 35992-8.

15. Basuroy S, Seth A, Elias B, Naren AP, Rao R. MAPK interacts with occludin and mediates EGF-induced prevention of tight junction disruption by hydrogen peroxide. Biochem J 2006;393(Pt 1):69-77.
16. Mannon P, Reinisch W. Interleukin 13 and its role in gut defence and inflammation. Gut 2012;61:1765-73.

17. Elliott SN, Wallace JL, McKnight W, Gall DG, Hardin JA, Olson M, et al. Bacterial colonization and healing of gastric ulcers: the effects of epidermal growth factor. Am J Physiol Gastrointest Liver Physiol 2000;278:G105-12.

18. Shin CE, Falcone RA Jr, Stuart L, Erwin CR, Warner BW. Diminished epidermal growth factor levels in infants with necrotizing enterocolitis. J Pediatr Surg 2000;35:173-6.

19. Hofmann GE, Abramowicz JS. Epidermal growth factor (EGF) concentrations in amniotic fluid and maternal urine during pregnancy. Acta Obstet Gynecol Scand 1990;69:217-21. 